Bol. Acad. peru. leng. 52. 2011 (139-157)

\title{
EL ÚLTIMO GARCILASO: LA ÉGLOGA III Y LA INCORPORACIÓN DE LA LITERATURA
}

\author{
Fernando Riva \\ Yale University
}

Fecha de recepción:

$13 / 11 / 2011$

Fecha de aceptación:

$15 / 11 / 2011$

La Égloga III escrita alrededor de $1536^{1}$ es el último poema de Garcilaso de la Vega. Como lo ha notado Rafael Lapesa, destaca entre toda la obra del toledano como una de sorprendente habilidad técnica, alcanzando casi niveles de perfección (159) tanto en la forma como en el contenido. Es la primera vez que utiliza la octava real, estrofa propia de la épica y que busca contar hazañas heroicas, con una maestría que le llama la atención al mismo Lapesa por su gracilidad y por superar la tendencia de esta estrofa a la monotonía (164). Asimismo, rompe con una larga tradición en la literatura pastoril en la cual la representación estaba basada en múltiples tópicos clásicos y en temas mitológicos. Si bien Garcilaso los utiliza al principio del poema, se distancia luego de ellos y se centra en su propia contemporaneidad a partir del río Tajo y de

1 La escritura de este poema se sitúa en 1536, fecha también en la que Garcilaso es herido de muerte en una campaña militar en el sur de Francia. Por tanto, la clausura de su ciclo poético es un hecho fortuito, donde su famosa divisa con la espada y con la pluma cobra dimensiones de realidad trágicas en la vida y en la obra del toledano. 
España, así como de la historia de amor entre Nemoroso y Elisa ${ }^{2}$. Todas estas innovaciones españolas se ubican en el relato de la ninfa Nise, razón de ser de la Égloga (Cammarata 53) en pleno centro del poema (Lapesa 149).

Esta novedad en la literatura de Garcilaso representa un quiebre en el marco de su obra y una madurez que se incorpora de forma plena en esta égloga. Este poema es un centro o una condensación exitosa de lo que Garcilaso había venido esbozando en sus anteriores composiciones y un planteamiento desafiante para la época. Buscaremos analizar la forma en la que la conciencia de su propia obra y del oficio de la literatura como actividad de representación artificiosa del lenguaje llega a su cénit, hasta integrar personajes desarrollados en otros poemas (como Nemoroso) y sus historias de amor (como la de este y Elisa) en diversos lugares de enunciación. Estas nuevas incorporaciones, sin embargo, no tendrían sentido sin la reutilización de los pasajes mitológicos de la obra, de los cuales se vale como instancias complejas de representación para otorgar actualidad al Tajo, a Toledo, a España, a Nemoroso y a Elisa. Estos se situarán más allá de la mitología y, más bien, se inscribirán en un mundo de ficciones literarias propias o -para utilizar un término propio de la época- en el de las fábulas. La cúspide de Garcilaso radica en literaturizar su presente, en ir más allá de los modelos antiguos, en dejar de lado un universo arcádico y, finalmente, en ofrecer a sus apreciadores la estetización de lo inmediato, es decir, de aquello que podían ver y tocar a la vez que leer. Así, nos enfrentaremos, luego de un complejo trayecto, no solo a la poesía de la inmediatez más allá de la mitología, sino a una surgida desde la originalidad de la literatura. Aquí, pues, radica la modernidad de Garcilaso.

2 No tomaremos como premisa los datos biográficos. Sin embargo, es moneda corriente en los trabajos de Rivers (264-5) y Lapesa (124) la consideración del personaje de Nemoroso como una proyección del Garcilaso enamorado de la dama portuguesa Isabel Freire, que para los críticos representa a la Elisa de sus obras. Si solo consideramos esta posibilidad, no solo Garcilaso actualizaría su ciudad, su río y España, sino su propia historia de amor, motor de toda su obra poética. 
La Égloga III está dirigida a María Osorio Pimentel, marquesa de Villafranca y esposa del virrey de Nápoles (Lapesa 159) en forma extensa. A lo largo de las siete octavas reales, metro quizá elegido para honrar a esta alta señora o a las hazañas no menos altas que se narrarán en este poema, Garcilaso reflexiona sobre los temas que lo han obsesionado durante su vida literaria: la fortuna, las adversidades, el oficio de poeta y el de soldado. Sin embargo, concentra todas sus fuerzas en dar testimonio de la inevitabilidad de la pluma poética y de lo obligado de su inclinación. Ya en la segunda estrofa de esta égloga, por ejemplo, escribe unos versos que serán canónicos en la literatura española ${ }^{3}$ :

$\mathrm{Y}$ aun no se me figura que me toca

aqueste officio solamente'n vida, mas con la lengua muerta y fria en la boca

pienso mover la voz a ti devida;

libre mi alma de su estrecha roca, por el Estygio lago conduzida, celebrando t'irá, y aquel sonido

hará parar las aguas del olvido (vv. 9-16, 419-20).

A partir de esta cita, no solo surge la conciencia del poeta acerca de su creación, que la corona como parte integral de su poética, sino que se genera una ambigüedad en el destinatario del texto. Es evidente que se dirige a María Osorio Pimentel, como él mismo lo declara; pero también surge la posibilidad, más sutil, de que lo haga a la memoria de Isabel Freire, vestida literariamente de Elisa. Sin buscar caer en las interpretaciones biografistas de la obra -como ya lo hemos declaradola dimensión autobiográfica de esta y el reflejo del amor del poeta son innegables en la literatura garcilasiana. Por eso, la alusión a la llegada del alma de este al Estigio, donde, para él, habita el olvido (y para la tradición, la muerte) nos pueden indicar la pista de un delicado juego de

3 Citaremos de la edición de Elías Rivers de las Obras Completas con comentario de Garcilaso de la Vega indicando la numeración de los versos que ocupan los fragmentos y luego el número de la página. Cuando se citen textos del propio Garcilaso distintos de la Égloga III, se consignará la obra antes de la numeración de los versos.

Bol. Acad. peru. leng. 52(52), 2011 
dobles destinatarios: por un lado la evidente María Osorio y, por otro, la ya difunta Isabel Freire en forma de una Elisa que inmortalizará en el texto. En este sentido, la visita al Hades por parte de un poeta que busca perpetuar a su amada acerca a Garcilaso a la figura de Orfeo (Correa 281, Cammarata 47) y a la dimensión del canto poético continuo. Con esto, confirma Garcilaso su papel dentro de la poesía, sobre la cual numerosas veces reflexiona, y, además, se autorrepresenta de modo velado a partir de un personaje mitológico, al cual abandonará hacia la mitad del poema. Es en este punto crucial donde se autorrepresentará como un creador concreto y cuyo centro será el paisaje del Tajo y de Toledo.

Hasta el momento, Garcilaso se muestra bastante cercano a su trayectoria anterior por sus elecciones mitológicas. Persiste en este hecho - por ejemplo- en la estrofa cuarta de la dedicatoria, después de reflexionar sobre las idas y venidas adversas de la fortuna, cuando se decide por completo a seguir escribiendo sobre el amor, encomendado a la tradición de Apolo y las nueve musas. Garcilaso no se dejará llevar por la fortuna y escribirá ayudado por estos, porque tiene un objetivo más loable y superior:

Pero por más que'n mí su fuerça prueve, no tornará mi coraçón mudable; nunca dirán jamás que me remueve fortuna d'un estudio tan loable; Apollo y las hermanas todas nueve me darán ocio y lengua con que hable lo menos de lo qu'en tu ser cupiere, que’sto será lo más que yo pudiere (vv. 25-33, 421).

En consecuencia, por más que la fortuna adversa, aun a través de la muerte, lo intente distraer de su empeño no lo logrará. Para seguir en su loable tarea de constante canto se entrega al ingenio de Apolo en una acción en la cual otorga su fuerza poética a las inspiraciones mitológicas. Más adelante este mecanismo, aunque crea mucha expectativa en el lector, se convertirá en un juego de atribuciones, equiparaciones y desatribuciones mitológicas para una creación independiente. 
Una vez declarados sus principios poéticos y planteados los finos mecanismos para lograr sus propios objetivos, el poeta manifiesta y resume su trayectoria, donde vida y literatura conforman una sólida unidad:

En tanto, no te offenda ni te harte tratar del campo y soledad que amaste, ni desdeñes aquesta inculta parte de mi estilo, que'n algo ya estimaste; entre las armas del sangriento Marte, do apenas ay quien su furor contraste, hurté de tiempo aquesta breve suma, tomando ora la espada, ora la pluma (vv. 33-40, 421-22).

En definitiva, las primeras estrofas de este poema se van convirtiendo no solo en un manifiesto literario, sino personal, donde las armas y las letras, es decir, donde Apolo, sus musas y Marte son los protagonistas y los motores de la vida del poeta. Es Garcilaso en forma sutil, un nuevo Orfeo que se autorrepresenta en función de estos dos dioses que constituirán simbólicamente el eje de su propia vida. Una vez identificado Garcilaso en estos términos, se encuentra listo para contar las cuatro historias de amor protagonizadas por cuatro ninfas en el marco de la propiciación y atribución mitológica. Así, el lector se ve guiado, en apariencia, hacia un Garcilaso más tradicional. Sin embargo, estamos muy cerca de la aparición de un elemento que gobernará el poema y que será la fuerza para despojarse de las identificaciones mitologizantes de la dedicatoria y del devenir de las historias: el río Tajo.

Gustavo Correa sitúa al río como el primero de los cuatro planos en los que se desenvuelve la égloga (279), es decir, como un elemento concreto y unificador. A partir de aquí, el escenario general será la vega toledana (Lapesa 159) matizada, en el mismo espíritu del principio, con los pasajes mitológicos. Quedará la mitología, en consecuencia, supeditada a la realidad del poeta, pero a una que el lector de entonces reconocerá también como suya. Esa es la grandeza de la égloga y la demostración de 
su madurez poética. Dice Garcilaso dando fin a la elegante y ambigua dedicatoria a la virreina:

Por aquesta razón de ti escuchado, aunque me falten otras, ser merezco; lo que puedo te doy, y lo que é dado, con recibillo tú, yo me 'nrriquezco. De quatro nymphas que del Tajo amado salieron juntas, a cantarme offrezco: Phillódoce, Dinámene y Climene.

Nise, que en hermosura par no tiene (vv. 49-56, 423).

En este momento, de manera explícita, aparece el Tajo de donde surgen las cuatro ninfas que contarán sus amorosas historias a través de sus tejidos. El río, entonces, es la fuente de aquellas y de esos amores doloridos. Lo interesante aquí es que en el momento preciso en el que los pronombres indicadores del destinatario del texto ("tú", por ejemplo, o "te") cesan, aparece de modo explícito el río Tajo, lo que nos podría indicar un posible vínculo entre la virreina, el Tajo y la literaturización de España en el contexto imperial, sobre el cual incidiremos más adelante. Garcilaso persiste en su proyecto de apartamiento de la mitología y creación de un conjunto de referencias de la realidad codificadas dentro de la literatura, cuando menciona a las cuatro ninfas y tan solo califica a Nise ("que en hermosura par no tiene"), quien será la encargada de contar la muerte de Elisa y difundir las historias de su trágico deceso no solo entre las otras ninfas, sino ante el propio Nemoroso. Nise posee, entonces, una hermosura única, porque a través de ella Garcilaso puede cambiarle el rumbo a toda su obra: salir de los modelos clásicos que tan bien manejó y retransformó, para proponer una literatura bastante más compleja.

De inmediato, y una vez terminadas las siete estrofas de la dedicatoria, pasa al terreno de la narración a través de los tejidos. Si en la octava anterior Garcilaso menciona explícitamente al Tajo, aquí consolidará su escenario y, por tanto, concretizará la noción de locus amenus. Leemos: 
Cerca del Tajo, en soledad amena, de verdes sauzes ay una espessura, toda de yedra revestida y llena, que por el tronco va hasta el altura y así la texe arriba y encadena que'l sol no halla paso a la verdura; el agua baña el prado con sonido, alegrando la yerva y el oýdo (vv. 57-64, 424-25).

El paisaje del Tajo es definido poéticamente en términos de un locus amenus. Sin embargo, lo que podemos ver en este punto es una equiparación del Tajo con toda la tradición clásica pastoril. Esto, creemos, no significa que toda la inclusión de elementos no mitológicos del poema se pueda explicar solamente en función de un ejercicio de equiparación con los clásicos que va desde el paisaje, nutrido por el aura del mito, hasta la dignificación inmortalizada y universal de la muerte de Elisa a la par de dioses y héores (Cammarata 54, Lapesa 162). Más bien, la equiparación cumple dos funciones. La primera es la construcción de un escenario palpable en términos clásicos para generar una dependencia final de las historias contadas por las ninfas a la realidad del Tajo, que generará el primer nivel mencionado por Correa, y el comienzo de la superación de la mitología a partir de una concreción inmediata. No se queda este hecho tan solo en la equiparación y dignificación del río en términos de literatura prestigiosa. Por el contrario, Garcilaso buscará otorgar brillo a la literatura desde sus paisajes españoles y las propias historias proyectadas.

Un segundo argumento de suma importancia es aquel de la complejización de los términos de representación. Al ser las historias contadas a través de tejidos, nos alejamos de una narración directa de estas en boca de las ninfas y nos acercamos a un segundo nivel de representación que podemos calificarlo como ecfrástico, vale decir, narración a través de la descripción literaria de la obra de arte. Aquí las obras de arte, es decir, los productos provenientes del artificio y de la técnica, son la mejor manera de narrar diferentes motivos también provenientes de los mismos ámbitos. Por ello, podemos hablar de la representación dentro de la 
representación o -en términos más claros- del arte dentro del arte. En el gran escenario retransformado del Tajo, hallamos una reflexión compleja de la mimesis artística en donde se contrasta una realidad concreta (pero literaturizada como la del río del poeta) y otra enteramente artificiosa como la de las historias en un segundo nivel de representación. Leemos:

\author{
Las telas eran hechas y texidas \\ del oro que el felice Tajo embía, \\ apurado después de bien cernidas \\ las menudas arenas do se cría, \\ y de las verdes ovas, reduzidas \\ en estambre sotil qual convenía \\ para seguir el delicado estilo \\ del oro, ya tirado en rico hilo (vv. 105-12, 430-1).
}

El Tajo provee el oro para entretejer las historias, es decir, los materiales pertinentes para este segundo nivel de representación. En consecuencia, se hace evidente el vínculo entre el río y el ejercicio de la mímesis ecfrástica con temas mitológicos. Así, si antes podíamos hablar de una equiparación, ahora podemos comprobar una relación de dependencia de la mitología hacia la rivera. La jerarquía se establece de la siguiente forma: el Tajo es el escenario principal en un primer nivel de representación, donde se sitúan, como puertas literarias o intermediarios, los tejidos constituidos por los propios materiales del río, hechos de oro que surge de la propia arena fina de aquel. El Tajo es, entonces, una entidad ganada ya per se y no a partir de la equiparación mitológica, válida en un principio tan solo como mecanismo retórico, y es asimismo la matriz de los problemas de la representación planteados por el poeta. Esta frontera mimética, observada en la construcción de los tejidos, es la actualización de las reflexiones presentes en la dedicatoria. La obsesión con lo obligado de su oficio y la declaración de sus esfuerzos encuentran su compleja razón de ser, en este punto en particular, mediante una reflexión general sobre la representación poética. No es gratuito, por tanto, que el poeta mencione el "delicado estilo" dado por el oro que le permitirá tejer las mejores historias de amor. Ya no son Apolo o las nueve hermanas las que propician la poesía en Garcilaso, sino el Tajo, 
río de su ciudad y centro de España. Toledo es el núcleo y Garcilaso el poeta que puede superar a todos los autores antiguos. Por eso, esta égloga es la cúspide de su trayectoria, porque es capaz de reflexionar sobre la poesía de forma directa como al principio de este poema; pero, además, puede representar literariamente esta reflexión a través de tal mecanismo ecfrástico. Todo aquí pertenecerá al territorio del artificio literario. Esta es la complejidad de la obra que actualiza en el artificio la literatura la explícita de la enunciación. Luego de igualar los dos planos, viene la desatribución mitológica o, mejor dicho, el otorgamiento de la propia identidad.

El sentido de la écfrasis, según Cammarata, radica en la posibilidad de poder proyectar los propios sentimientos con la distancia que le proporciona esta representación. Se distancia, entonces, de la enunciación directa para observar mejor el reflejo de sus pesares amorosos en estas pinturas mitológicas (50). En efecto, se distancia de ellas y las coloca en primer plano -otra vez- sin la voluntad de elevar la suya propia, sino para utilizarlas como peldaños poéticos para luego abandonarlas e incluso rechazarlas indirectamente. Solo las usa para aderezar la historia de fondo de Elisa y Nemoros, y agregarle el marco indicado y verosímil de la muerte y la tragedia reflejada en la historia de Orfeo y Eurídice, la de Apolo y Dafne y, finalmente, la de Venus y Adonis (Cammerata 49-51). Todas se engarzan a partir de los tejidos de las ninfas con la propiciación otorgada por el Tajo. Por último, en el marco de la construcción de esta nueva mímesis ecfrástica se insiste en la tradición clásica ya no referente a la poesía, sino a la pintura. Garcilaso cierra su compleja introducción a este ejercicio otra vez atribuyendo a los pintores antiguos la maestría de lo que en estas telas las ninfas tejen:
La delicada estambre era distinta
de las colores que antes le avían dado con la fineza de la varia tinta que se halla en las conchas del pescado; tanto arteficio muestra lo que pinta y texe cada nympha en su labrado 
quanto mostraron en sus tablas antes

el celebrado Apelles y Timantes (vv.113-120, 430-31).

Esta atribución mitológica obedece a propósitos retóricos que apuntan a mantener tres largas historias mitológicas. Garcilaso tiene preparado el terreno para el despliegue del tejido de Filódoce, Dinámene, Climene y, sobre todo, de Nise. El toledano es el artífice que crea esta égloga en un primer nivel de la enunciación, de la misma manera que lo son las cuatro ninfas que mostrarán las cuatro historias. Garcilaso compone poesía y en ella escribe que las ninfas pintan fábulas de amor. Así, el poeta escribe de otros artífices en su proceso de creación. Aquí, por ejemplo, radica uno de los niveles de complejidad de esta obra.

Ya había intentado la representación ecfrástica en la Égloga II cuando Nemoroso, el mismo que aparecerá representado en este texto, narra la historia de Severo quien, en el río Tormes, halló una urna que contaba las grandes hazañas de la casa de Alba. Leemos:

A aquéste el viejo Tormes, como a hijo, le metió el escondrijo de su fuente, de do va su corriente començada; mostróle una labrada y cristalina urna donde'l reclina el diestro lado, y en ella vio entallado y esculpido lo que, antes d'aver sido, el sacro viejo por devino consejo puso en arte, labrando a cada parte las estrañas virtudes y hazañas de los hombres que con sus claros nombres ilustraron cuanto señorearon de aquel río (Égloga II vv. 1169-80, 375-6).

Aquí Garcilaso aprovecha la localidad de Alba de Tormes, a cuyos duques alude aquí, para hablar sobre otro río español. Lo curioso es que este concentra el espacio para el hallazgo de la urna y reúne el primer ejercicio de representación ecfrástica, de donde surgen hazañas heroicas. Aquí puede haber un esbozo inicial que logra su plenitud en los tejidos 
de las musas, de donde surgen historias de amor. De alguna manera, ambas écfrasis conducen a los temas constitutivos del sujeto Garcilaso: el de las armas y el de las letras, y sugieren que la forma en que estos dos dominios se expresan es la del artificio, único camino, a su vez, para la autorrepresentación del propio Garcilaso. Los lectores sabemos de él por los dos ejes de su vida y estos, a su turno, encuentran su plena actualización literaria, su fabulización más exitosa, en los momentos de una compleja representación cimera en las écfrasis. En la égloga segunda habla de las hazañas de los nobles; en la égloga tercera, de cuatro historias de amor, donde se encuentra la representación de la suya. Garcilaso, así, se refleja en estos textos en un conjunto de juegos especulares, donde la verdad de su biografía se llena de artificio y se expande entre historias contadas por urnas halladas por un pastor o, más elaborada, honesta y vívidamente, por ninfas que tejen mantos.

En consecuencia, Garcilaso logra en ambas églogas no solo una familiaridad de motivos, expresada al presentar tanto al Tajo de la tercera como al Tormes de la segunda en el papel de propiciadores de la representación literaria compleja, sino que sitúa a un personaje particular, Nemoroso, vinculado de una manera u otra con este tipo de mecanismos. Tenemos, por tanto, personajes y técnicas que se repiten; pero, sobre todo, que se perfeccionan desde la segunda hasta la tercera, donde se universalizan y canonizan a partir de la literatura.

Una vez contadas las historias de Orfeo y Eurídice, de Apolo y Dafne, y de Venus y Adonis, el terreno está preparado para que la hermosa Nise cuente la suya. Dice Garcilaso:

La blanca Nise no tomó a destajo de los pasados casos la memoria, y en la lavor de su sotil trabajo no quiso entretexer antigua historia; antes, mostrando de su claro Tajo en su labor la celebrada gloria, la figuró en la parte donde'l baña la más felice tierra de la España (vv. 193-200, 438). 
Hemos llegado al núcleo de la égloga. Queda claro que la equiparación del Tajo con el paisaje ameno clásico fue solo un instrumento para su lanzamiento al primer plano de la creación literaria de la poesía y que el conjunto de atribuciones estaban destinadas a preparar el terreno para que la historia de Nise brille por sí sola como creación por completo poética y original, y no como recreación de la mitología. Esta, acaso, a lo largo de las tres anteriores, ha servido para contrastar el carácter especial de la historia de Nise. Por eso, la ninfa "no toma a destajo" los mitos y decide contar, por su propia voluntad, desde el glorioso río, una historia que surgió del que "baña la más felice tierra de la España". Este es el hecho más tajante de desatribución mitológica. A partir de aquí el poema cambia y se convertirá en la cúspide de la obra del poeta. Nunca más, en todo el texto, se volverá a mencionar una "antigua historia", a pesar de que las ninfas aparezcan lamentándose por la muerte de Elisa. Todo lo que viene es la literaturización de la realidad y su proyección en la posibilidad de la fábula. La mitología ahora estará al servicio de la creación original tanto en forma como en contenido.

La conciencia de centralidad del Tajo en España y, consecuentemente, en la gloria del Imperio (de allí nuestra acotación según la cual la dedicatoria de la obra a la virreina de Nápoles puede aludir a ella) se traduce también en la centralidad de la égloga. Se comprueba, de nuevo, que no se dignifica al Tajo como parte de un contexto mitológico, sino que se lo eleva como el glorioso centro de la mejor tierra de España, en opinión del poeta. Por esa razón, Garcilaso utiliza las octavas reales, para contar las hazañas del amor glorioso que el Tajo, principal propiciador de la poesía y centro de la enunciación garcilasiana, inspira en la tela de Nise. El amor y la muerte de Elisa, entonces, son hechos protagonizados por héroes literarios de la imaginación de Garcilaso o de su proyección personal. En cualquier caso, sin embargo, son parte de su propia literatura, con un Nemoroso que mira las telas y una Elisa llorada antes ya en la égloga I. Estas historias de por sí, además de las glorias del río y de España, son dignas de ser contadas en octavas reales. El lugar central de Toledo, ciudad del poeta y paisaje principal del Tajo, también se menciona en la égloga. Nise, desde lo alto, observa la ciudad: 
Estava puesta en la sublime cumbre

del monte, y desd' allí por él sembrada, aquella illustre y clara pesadumbre

d'antiguos edificios adornada.

D'allí con agradable mansedumbre

el Tajo va siguiendo su jornada

y regando los campos y arboledas

con artificio de las altas ruedas (vv. 209-16).

La ciudad de donde se enuncia es ilustre y está adornada por importantes y antiguos edificios. Este preciso hecho, entonces, explica el abandono de la mitología y la opción por la realidad concreta de la ciudad y del río. Aquella ya no es necesaria para elaborar su obra ni para cubrirla con el manto del prestigio. Toledo y el Tajo (y, por tanto, España y el Imperio) serán lo suficientemente grandes y gloriosos para albergar la sublime historia de la muerte de Elisa y del sufrimiento de Nemoroso. La antigua ciudad amurallada deja paso al manso Tajo que riega los campos como señal de abundancia. Ya no es, empero, una riqueza de la áurea edad, sino aquella fundada en la gloria de España. Otra vez se actualizan los dos ejes de la obra del poeta en una compleja disposición: por un lado, la ciudad que preside, amurallada y poderosa como señal de las armas y del Imperio al que Garcilaso sirvió como soldado; por otro, el río que, manso, da vida y plenitud, y sobre el cual escribió como poeta. Este es el nuevo marco desde el cual hará hablar a sus propias creaciones literarias.

La reaparición de las ninfas llorando por la muerte de Elisa -ahora una de ellas- no es una mitologización para ensalzarla y erigirla en inmortalidad universal, a partir de la continuidad entre la historia de Eurídice y de Elisa (Correa 279-81). Es, más bien, un proceso complejo de "garcilacización" de la mitología, en donde -como vimos- ella ahora solo es instrumento de expresión. Esta es la cima de su madurez y de su poesía:

Todas, con el cabello desparzido, lloravan una nympha delicada cuya vida mostrava que avia sido 
antes de tiempo y casi en flor cortada; cerca del agua, en un lugar florido, estava entre las yervas degollada qual queda el blanco cisne quando pierde la dulce vida entre la yerba verde (vv. 225-32, 440-41).

La mitología, entonces, es solo un material con el que cuenta Garcilaso sobre la base de una expresión bien fundada en todo el trabajo de elaboración que hemos visto a lo largo de la égloga. El desarrollo del tema de la muerte es, en este sentido, la actualización esplendorosa de lo que había venido intentando hacer desde la égloga primera, donde Nemoroso lloraba, en forma directa, la muerte de la amada:

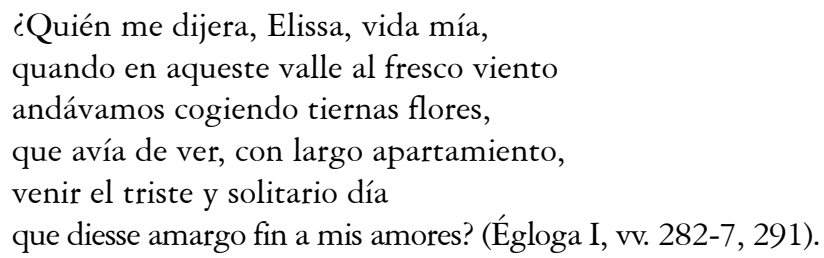

Lo que prima en el pasaje de la égloga primera es el recuerdo expresado -como dijimos- en forma directa en boca de Nemoroso. Acaso lo que quiere Garcilaso, en contraposición con el texto anterior, es distanciarse del dolor del pastor poeta (quizá una representación de sí mismo) a través de la elaboración literaria de la muerte de Elisa, por medio de la écfrasis en la égloga tercera. Así, sitúa él la muerte de la amada en el ámbito de lo puramente estético, donde eleva el probable plano personal de este hecho literario a una esfera donde el dolor halla una forma universal (Correa 281). En efecto, esta es, de forma plena, la inscripción de Elisa en la fábula literaria más pura, en la elaboración poética perenne a través de la historia contada por un tejido. De la misma manera que lo ocurrido con Toledo y el Tajo, Elisa ingresa aquí al puro universo poético al punto que yace, en innovadora forma, degollada sobre la yerba. Garcilaso, como todo gran creador, va más allá de su historia personal. El amor, en consecuencia, motor de la literatura del toledano, queda grabado en la tela para el poeta, para sus personajes y 
para los lectores. El tema de la flor arrancada, metáfora de lamento en la égloga primera, es imagen literaria en écfrasis no solo en el parnaso personal de aquel, sino en el novísimo parnaso español de entonces. El distanciamiento artificioso del amor es una cumbre también de lo que Garcilaso consigue aquí.

Ya distanciado a partir de la literaturización de Elisa, es capaz de hacerla hablar por primea vez en discurso directo. Si antes los pastores eran los que hablaban de ese modo, ahora lo es Elisa, causa de la queja de Nemoroso. Este desplazamiento revela la madurez del poeta en el manejo de sus creaciones literarias por el distanciamiento artificioso visto antes. Cada personaje está tan bien creado que es capaz aquí de hablar por primera vez como la amada o como en el caso del elocuente Nemoroso, de no demostrar sus quejas, eje fundamental de la égloga primera. En el marco del desarrollo pleno de la écfrasis, la tela de Nise nos cuenta que una bella pero triste diosa escribe el epitafio de Elisa sobre la corteza del árbol:
"Elisa soy, en cuyo nombre suena y se lamenta el monte cavernoso testigo del dolor y grave pena en que por mí se aflige Nemoroso y llama 'Elisa'; 'Elisa a boca llena responde el Tajo y lleva pressuroso al mar de Lusitania el nombre mío, donde será escuchado, yo lo fío".

En este preciso momento, la écfrasis se complica muchísimo, pues la descripción de las telas a través de las cuales se cuentan las historias ahora nos muestra la voz de Elisa mediante su epitafio inscrito en el texto con la forma de un poema; en otros términos, la expresión poética se reelabora de tal modo que la voz está simulada por la escritura dentro de la representación plástica general. Este es un artificio literario en donde los varios niveles de representación cobran un mismo nivel y se equipara palabra con imagen, pero siempre con la conciencia de que la voz directa o la queja viva no son posibles sino en el marco del artificio. Así, las 
imágenes son palabras y viceversa: solo eso. Este es el manifiesto poético donde la realidad de las historias se recrea exitosamente dentro de otras representaciones. No solo esto es la incorporación y consolidación de Elisa como personaje literario, sino la de toda la literatura de Garcilaso dentro de un poema propio. Esta es la realización de un juego de espejos, reflejos y representaciones que mencionamos al principio.

Si interpretamos el contenido del epitafio, distinguimos que Elisa es consciente en forma plena de sí, pues se presenta por nombre y da cuenta de sus características como el dolor y la pena y, además, llama a Nemoroso, su amado pastor cuyas quejas conocemos de sobra. La historia de amor se cierra aquí, sin necesidad de mitología. Sin embargo, es este un amor trágico, porque el reconocimiento del amado se da precisamente en la lectura del epitafio, es decir, en el momento en el que ya Elisa yacía degollada y muerta. La voz de Nemoroso es mucho más compleja aquí que la simulada voz de Elisa en la escritura. Es paradójico que este personaje, caracterizado por su explayada expresión en cientos de versos tanto en la égloga primera como en la segunda, únicamente diga una palabra en una voz artificial. Es como si este epitafio fuera el resumen de toda la obra de Garcilaso y como si bastara la expresión del nombre de la amada en un texto de la propia amada para cifrar todo su sentimiento. Esta es la militancia de amor de Nemoroso y la dependencia de este respecto de la amada. Es el mejor homenaje de amor de Nemoroso a Elisa y de ella a él. Se va creando una cadena y una explicación para toda la literatura anterior de nuestro poeta: el sufrimiento de un personaje cifrado en un nombre y la representada voz de ella dirigiéndose al pastor. Así tenemos a Elisa y a Nemoroso, pero también al río Tajo en prosopopeya. Este río central en la propiciación de la poesía se ha incluido dentro de la poesía participando de la historia de amor. La voz del río surge en el epitafio, pronunciando el nombre de la amada de Nemoroso. El río de Toledo en toda la fertilidad de su potencia, en pleno centro de España y al lado de la ciudad amurallada y antigua, pasa de ser una figura real y palpable -como hemos visto- a ser un partícipe parco, pero suficiente al igual que Nemoroso. Si antes el paisaje del Tajo era equiparado para cobrar su validez e independencia literaria como espacio, ahora es un personaje más, con lo que se llega al ápice de su fabulización y de su 
incorporación literaria. La cadena queda trazada entre la Elisa muerta, el parco Nemoroso y el Tajo, cuyo caudal llevará el nombre de Elisa por todo su cauce hasta el mar de Lusitania. El río conduce las palabras anunciando los amores junto con la grandeza de Toledo e irá siempre, llevando literatura, más allá, tal como la divisa imperial sostiene. Este es el núcleo final y probablemente el inicio del afán poético de Garcilaso: expandir su voz poética y servir al Imperio.

Si bien las historias de los héroes y dioses de las tres ninfas anteriores preparan el terreno para esta tragedia, son solo anuncios y no equiparaciones. Garcilaso no engalana su literatura con ellas, sino que ellas están al servicio de esta historia solo como antecedentes, como muestras de la tragedia máxima del amor que no será aquí el mito de Orfeo y Eurídice, sino el cuento de Nemoroso y Elisa (o el de Garcilaso e Isabel). He aquí otra vez el centro de la representación y el ejercicio consciente y maduro de toda su reflexión sobre su oficio de creador.

Nemoroso en su dimensión individual ya es un personaje de forma clara, incorporado y reinterpretado, al punto que el poeta lo coloca como un espectador de las historias que cuenta la tela de Nise:

En fin, en esta tela artificiosa toda la istoria estava figurada que en aquella ribera deleitosa de Nemoroso fue tan celebrada, porque de todo aquesto y cada cosa estava Nise ya tan informada que, llorando el pastor, mil vezes ella se enterneció escuchando su querella (vv. 249-56, 444).

Nemoroso ha cobrado tal independencia como sujeto que es el espectador de su propia historia. Así, es un espectador más que observaba la "tela artificiosa" de Nise cuyo cuento lo movió a las lágrimas. No solo se vio reflejado en la cúspide de su amor pronunciando el nombre de su amada en el juego de las representaciones, sino que estas lo llevaron a las lágrimas. Esta es la demostración más sólida de la eficacia y del éxito 
de las ficciones de Garcilaso. Salimos, entonces, del nivel ecfrástico para encontrarnos de nuevo con un río Tajo que es el espacio de los pastores que ya no cantan sus penas, sino que lloran sus propias historias vistas y oídas. Este es el giro fundamental de la poesía de Garcilaso: los que antes cantaban y eran protagonistas ahora oyen y se conmueven. Esta es, de manera exacta, la definición del funcionamiento de la literatura en general en tan solo un poema a partir de un material propio.

Finalmente, se cierra la historia de la tela de Nise con la final canonización de los amores de Elisa y Nemoroso: su difusión. Esta no debe quedar en el espacio de las selvas ("y porque aqueste lamentable cuento / no solo entre las selvas se contasse" vv. 257-8), es decir, de la literatura de pastores, sino que debe, como el nombre de Elisa llevado por el Tajo en su epitafio, ir más allá ("quiso que de su tela el argumento / la bella nympha muerta señalasse / y ansí se publicasse de uno en uno / por el húmido reyno de Neptuno" vv. 261-4). En otras palabras, esta fábula se expande, pues concentra el funcionamiento de la representación literaria; sale del universo pastoril; y lleva, asimismo, estas ficciones españolas, con el Tajo y Toledo como centro, a todos los mares a la manera del Imperio.

De nuevo, surge el Garcilaso que empuña ora la espada, ora la pluma. Su labor literaria está culminada en la dignificación de la España imperial y en la creación de una literatura propia, a partir de sus mismas ficciones, donde la renuncia de la mitología era un momento obligado. Así, Garcilaso deja de lado la mitología aun propia de España, las columnas de Hércules, por ejemplo, y opta por la canonización y representación de su conciencia literaria. Lo que sigue después en el poema es el diálogo entre Alcino y Tirreno. Vuelve Garcilaso a los pastores, pero sin angustia en el deleite de la pura literatura que vale por sí misma, pues ya explicó y cifró su funcionamiento. Los amores son relatados sin angustia en la forma directa tradicional. Otra vez, nos encontramos en un universo lleno de tópicos, pero desde el sosiego de la pura representación. Por eso, se da el lujo de volver a los cánones pero, de ninguna manera, al pasado. A Garcilaso ya no lo gana más la quiebra, sino el hallazgo de la literatura. 


\section{BIBLIOGRAFÍA}

CAMMARATA, Joan. Mythological Themes in the Works of Garcilaso de la Vega. Madrid: Porrúa, 1983.

CORREA, Gustavo. "Garcilaso y la mitología”. Hispanic Review, 45, 1977, pp. 269-81.

GARCILASO DE LA VEGA. Obras completas con comentario. Edición crítica de Elías Rivers. Madrid: Castalia, 1974.

LAPESA, Rafael. "La trayectoria poética de Garcilaso”. Garcilaso: Estudios completos. Edición corregida y aumentada. 3ra edición. Madrid: Bella Bellatrix-Istmo, 1985, pp. 9-176.

\section{Correspondencia:}

\section{Fernando Riva}

Docente de la Universidad de Yale. 